\title{
A PET-positive rapidly growing mass of the abdominal wall after cesarean section with an unexpected diagnosis of vernix caseosa granuloma: a case report
}

\author{
Antonio Maccio ${ }^{1 *}$, Paraskevas Kotsonis ${ }^{1}$, Fabrizio Lavra ${ }^{1}$, Giacomo Chiappe ${ }^{1}$, Ester Mura ${ }^{2}$, Luca Melis ${ }^{3}$ \\ and Clelia Madeddu ${ }^{4}$
}

\begin{abstract}
Background: Abdominal wall tumors are rare and include heterogeneous diseases. Among them, desmoid tumors are the most frequent and are often diagnosed in young women during or early after pregnancy; inflammatory response after trauma or microtrauma, such as after cesarean section, may favor their growth.

Results: A 37-year-old woman presented with a progressive mass in the abdominal wall after a cesarean section. Positron emission tomography imaging confirmed a positive mass with a high maximum standardized uptake value; a biopsy suggested a myofibroblastic tumor. With continued tumor growth and worsening symptoms, the mass resembled a desmoid tumor; therefore, we proceed with its resection. The final diagnosis was foreign body granuloma as a reaction to the spillage of meconium and keratinous material in the amniotic fluid during cesarean section.

Conclusions: The present case provides information on an abdominal wall foreign body granuloma arisen from meconium and vernix caseosa after cesarean section, which presented an atypical clinical picture, mimicking a desmoid, thus requiring a careful diagnostic and treatment approach.
\end{abstract}

Keywords: Desmoid tumor, Foreign body granuloma, Cesarean section, Sarcoma, Meconium, Vernix caseosa

\section{Background}

In women, tumors of the anterior abdominal wall are rare and encompass very different pathologies, most commonly soft tissue sarcomas (mainly fibrosarcomas, dermatofibrosarcoma protuberans, and liposarcoma) and aggressive fibromatosis or desmoid tumors [1]. The differential diagnosis of abdominal wall tumors in women includes also sarcomatoid dissemination (particularly after conservative surgery in presence of occult leyomiosarcoma), metastases, or endometriosis implants [2]. Among these rare pathologies, desmoid tumors are the most frequent. They are very difficult to discriminate from sarcomas since they may present overlapping clinical behavior and radiological characteristics [2]. In fact,

\footnotetext{
* Correspondence: a.maccio@tin.it

${ }^{1}$ Department of Gynecologic Oncology, Azienda Ospedaliera Brotzu, via

Edward Jenner, 09121 Cagliari, Italy

Full list of author information is available at the end of the article
}

rapid growth and pain represent the more typical symptoms of both these conditions. Notably, desmoids may develop in women of fertile age, especially during pregnancy and puerperium [3]. In addition, some abdominal wall sarcoma that developed in pregnancy has been described, particularly cases of dermatofibrosarcoma protuberans [4]. Even abdominal wall endometriosis may be related to pregnancy since it typically localizes at the surgical incision, such as cesarean section scars [5], and is associated with progressive pain.

Here, we describe a very rare case of a voluminous foreign body granuloma consequent to the spillage of meconium and keratinous material in the amniotic fluid developed in the abdominal wall after a cesarean section in a woman with previous myomectomy with morcellation for an atypical leyomioma. 


\section{Methods}

The data of this case report was obtained through retrospective chart review. Written informed consent was obtained from the patient for the publication of the case report and the accompanying images.

\section{Results}

A 37-year-old woman (gravida 2, para 1) presented with a painful mass in the abdominal wall 1 month after a cesarean section delivery. Physical examination revealed a 3-4-cm palpable mass, which grew progressively with even more persistent pain; 15 days after the first check, the mass occupied all the lower quadrants of the abdominal wall. With the patient having a history of abdominal myomectomy for a large uterine leiomyoma (350 g, mitotic index 5/10 HPF; focal moderate atypia; Ki67, 15\%) 2 years before, we hypothesized various possibilities based on our previous experience [6]: (a) recurrence of the previous myoma, (b) sarcomatoid degeneration of residuals of previously excised myoma, or (c) a desmoid tumor. Then, we performed computed tomography (CT), positron emission tomography (PET), and ultrasound-guided biopsy. CT showed an irregular formation in the lower part of the rectus muscles, consisting of a hyperdense ring that enclosed a hypodense area. This mass bordered in the adipose tissue on the anterior abdominal wall. To better assess its characteristics, etiology, and malignancy, the patient underwent PET/CT, which showed a large, irregular, and inhomogeneous high ${ }^{18} \mathrm{~F}$-fludeoxyglucose uptake (maximum standardized uptake value [SUV], 12.9) in the anterior abdominal wall. This area seemed to extend beyond the muscle tissue, possibly involving the omentum (Fig. 1). Histological examination of the Tru-cut needle biopsy showed loose connective tissue consisting of irregularly intersecting bundles of blended cells with modest nuclear atypia, intensely infiltrated by lymphoplasma cells (smooth muscle actin+/-; vimentin+; beta-catenin-; desmin-; Ki67 5\%), suggesting a myofibroblastic tumor.

The mass continued to grow, and the associated symptomatology became increasingly worse and debilitating. The patient agreed to undergo surgical removal of the neoformation. Based on PET report, we started performing a diagnostic laparoscopy. After observing no connection between the mass and the abdominal cavity, we proceeded with a pubo-umbilical laparotomy and pointed out a voluminous yellow neoformation that started from the anterior sheath of the rectus muscles and extended to the subcutaneous adipose tissue (Fig. 2). We incised the sheath by involving approximately $2 \mathrm{~cm}$ of peripheral normal tissue using the LigaSure ${ }^{\text {тм }}$ Small Jaw Open Sealer/ Divider (Covidien, CO, USA). A good cleavage plan was found between the sheath and the rectus muscles, which were thus spared by excising the neoformation with the electric scalpel. The intraoperative histologic examination excluded a malignancy. The mass weighed approximately $300 \mathrm{~g}$ and measured $11 \times 10 \times 4 \mathrm{~cm}$ (Fig. 3). A biological mesh was applied. Final pathologic examination revealed the presence of striated muscular cells, a myofibroblastic

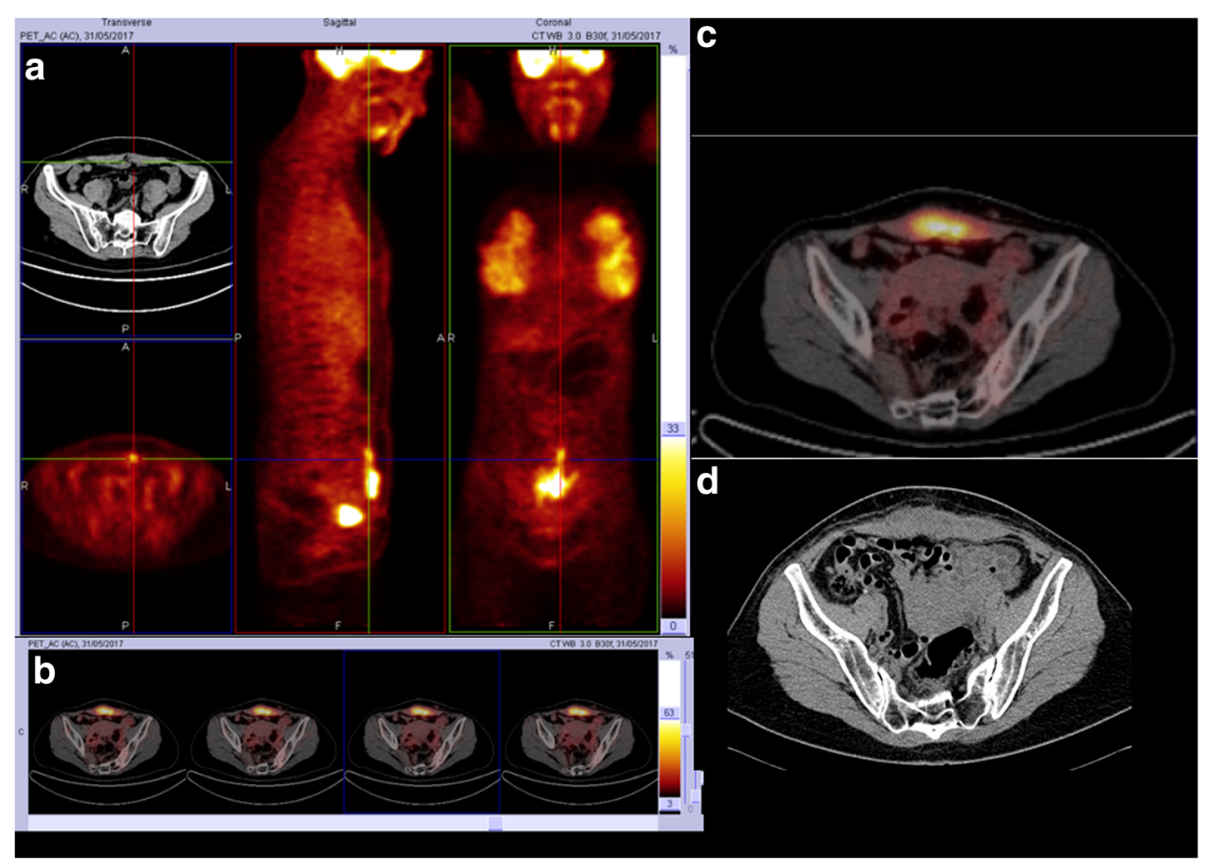

Fig. 1 Preoperative PET (a-c) and CT (d) scan showing the neoformation located in the in the context of the lower part of the rectus muscles of the abdomen. The PET showed an area of irregular and inhomogeneous hypercaptation in the context of the formation of the anterior abdominal wall with a very high maximum standardized uptake value (SUV max up to 12.9) 


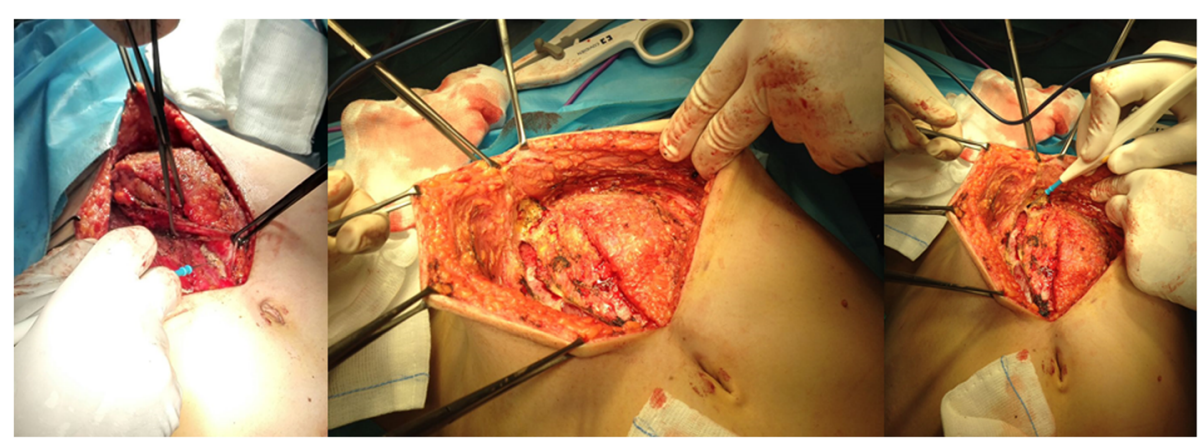

Fig. 2 Intraoperative pictures of surgery for abdominal wall neoformation. The mass started from the anterior sheath of the rectus muscle of the abdomen and extended to the adipose tissue of the subcutaneous layer

proliferation with giant cells resulting from foreign body reaction, granulocytes that infiltrated the adipose tissue (more typically present near sutures or centered on fetal squamous cells aggregates and keratin debris as observed in amniotic fluid (vernix caseosa), amorphous material compatible with meconium, and necrotic areas (Fig. 4). Immunohistochemistry showed positivity for smooth muscle actin and negativity for beta-catenin. Molecular analysis revealed a wild-type beta-catenin gene. Surgical margins were tumor-free. The final histological diagnosis was a foreign body granuloma.

To better characterize the inflammatory component of the neoformation, immediately after surgery, the cells obtained from mechanical and enzymatic degradation of the fresh tumor sample were separated by a discontinuous double density gradient (75-100\% Ficoll-Hypaque), and their phenotype assessed by flow cytometry (Accuri C6, BD Biosciences, Erembodegem, Belgium) [7]. The cells isolated in the $75 \%$ gradient were threefold higher than those isolated in the $100 \%$ gradient. In the $100 \%$ gradient, cells were mainly activated $\mathrm{T}$ lymphocytes (CD3+/CD4+/Glut+, 47.6\%; CD3+/CD8+/Glut+, 31.3\%). Macrophages (CD14+) were the predominant cells $(70 \%)$ in the $75 \%$ gradient; they were mostly M1 (CD14+/CD80+/Glut+, 48.9\%) with less M2-polarized cells (CD14+/CD163+/Glut-, 20.4\%) (M1/M2 ratio, 2.4). Additionally, in the $75 \%$ gradient, we found mesenchymal stem cells/fibrocytes (CD13+/CD14 -/Glut-, 13\%; CD10+/CD33+/CD14-/Glut-, 10\%).
Seven days following drainage removal, the patient presented with a subcutaneous effusion positive for Klebsiella pneumoniae. This required a percutaneous drainage within the fluid collection and infusion of $200 \mathrm{mg}$ vancomycin in $10 \mathrm{~mL}$ saline solution through the drain twice a day for 7 days [8-10]. Since the problem did not resolve, we removed the mesh and used a medication with a single-use negative-pressure wound therapy system (PICO, Smith and Nephew, Hull, UK). Seven days after the removal of the wound therapy system, the patient was in optimal condition. Six months after surgery, she was disease-free.

\section{Discussion}

Tumors of the anterior abdominal wall constitute a rare class of neoplasia. A very important aspect of this clinical case is the unpredictability of its onset, making it unique and rare, being the case of a rare condition mimicking another rare conditions hence the need to describe it. In fact, the incidence of abdominal soft tissue sarcoma is about 2.8 cases per million per year (less than $5 \%$ of all soft tissue sarcomas) [1] similar to that of desmoid tumors, i.e., 2-4 cases per million per year [11], and the present case seems to be the first described in the literature. Desmoids preferentially develop in women of fertile age, especially during pregnancy and in puerperium. Hormonal factors such as pregnancy-related hyperestrogenism may favor their formation [3]. Since

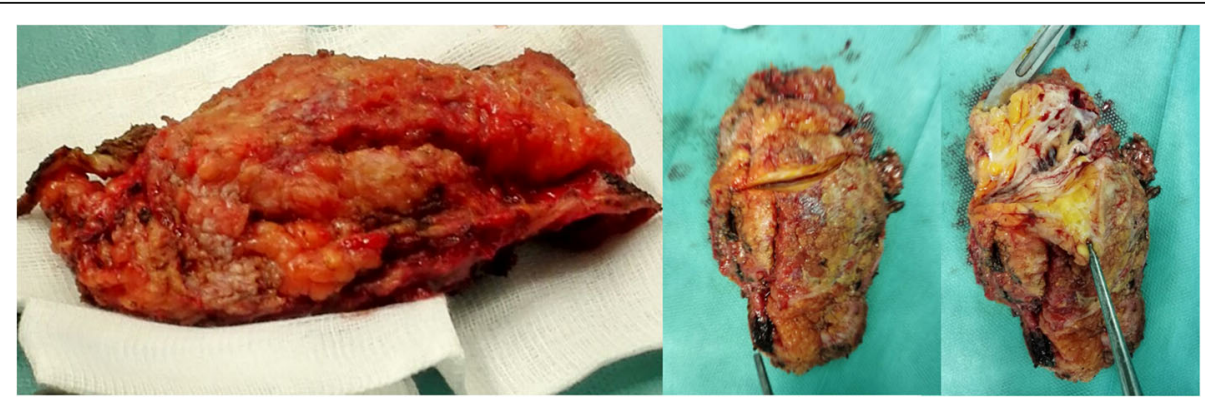

Fig. 3 Macroscopic view of the excised abdominal wall mass weighing approximately $300 \mathrm{~g}$ and sized $11 \times 10 \times 4 \mathrm{~cm}$ 


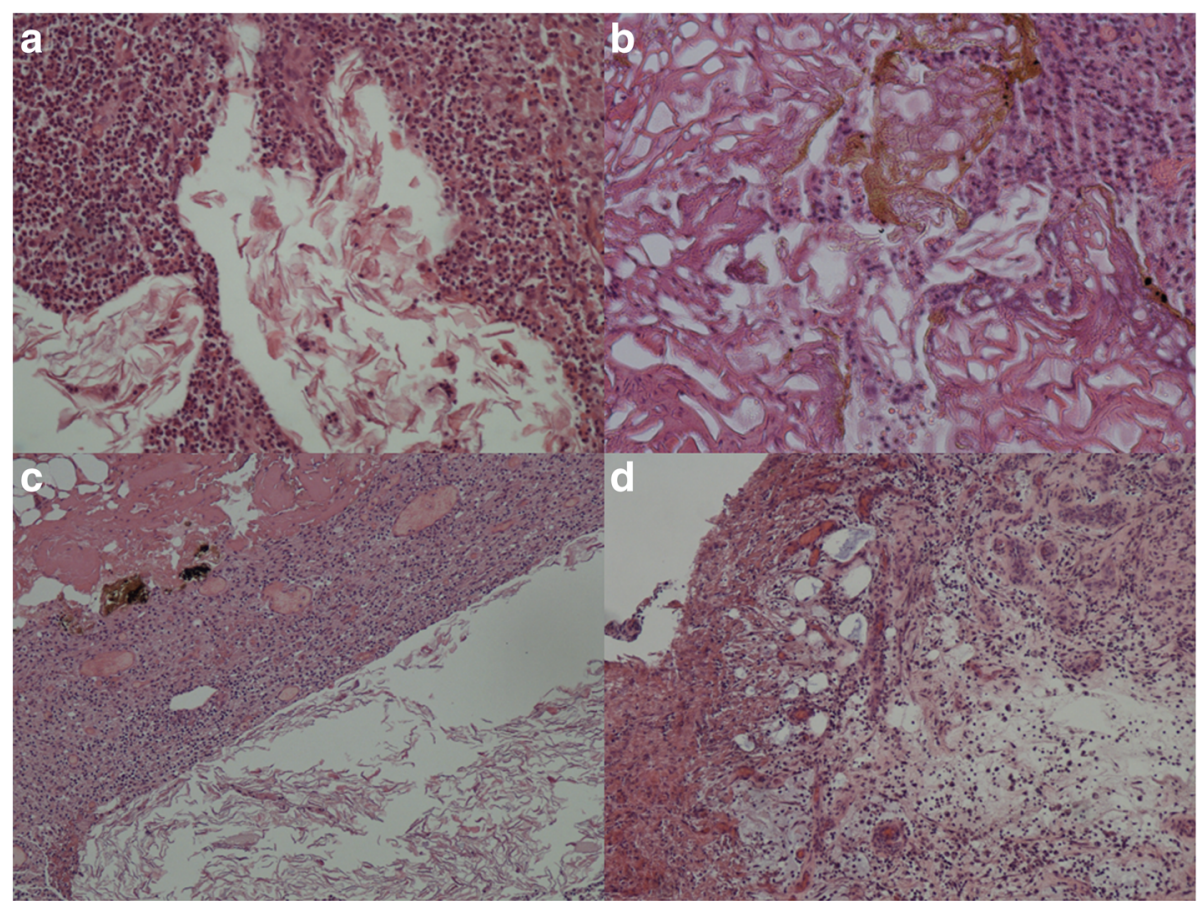

Fig. 4 Histological exam of the excised neoformation. a Keratin debris mixed with giant cells as for foreign body reaction (hematoxylin-eosin). b Keratin debris and meconium accompanied by inflammation that dissociates intensely edematous muscle tissue (hematoxylin-eosin). c Keratin debris surrounded by acute purulent inflammation, in the upper left amorphous brownish material referable to meconium (hematoxylin-eosin). d Granulation tissue and edema with evident neovascularization and presence of multinucleated giant cells (hematoxylin-eosin)

they may arise more frequently in the puerperium, traumas or microtraumas to the abdominal wall with consequent tissue correction may cause their onset [3]. Furthermore, surgery and inflammation may induce tumor growth. Typically, wound healing is a strictly controlled, self-limited process of tissue regeneration and remodeling. In response to tissue injury, mesenchymal cells are recruited to promote wound healing [12]; these pluripotent cells differentiate into fibroblasts, myofibroblasts, and endothelial cells. Therefore, the phenomena of repair, cicatrization, correction, and healing may lead to a series of events causing desmoid tumors, especially in people with a genetic predisposition to beta-catenin deregulation [11].

The present case showed a completely different clinical phenomenon. Although the symptomatic and growth characteristics of the neoformation resembled those of a desmoid tumor, the PET findings were positive, with a very high SUV. This influenced our presurgical diagnosis. In fact, desmoids are unlikely to be PET positive due to their low mitotic index, and if they are (but rarely), the SUV is very low [13]. One could also hypothesize a sarcoma caused by the sarcomatoid degeneration of residuals of the previous myomectomy. When a myomectomy is performed in confined spaces with the need for morcellation, the spillage of tissue components may occur, which over time, following adequate stimuli, may evolve into disseminated myomatosis and, in more serious cases, sarcomatoid degeneration
[6]. However, in our case, the preoperative biopsy did not confirm the suspicion of sarcoma but rather reinforced the hypothesis that it could be an atypical desmoid due to its high SUV index. According to the international guidelines [1], we performed a total surgical excision of the neoplasm. The definitive histology showed an extremely complex and rare problem; the desmoid nature was not confirmed (the lymphomonocytic component was prevalent, beta-catenin was negative, and the beta-catenin gene was wild type), but a foreign body granuloma was diagnosed. The foreign body consisted of amniotic fluid and meconium, suggesting either an inadequate cleaning of the cesarean incision site during suturing or, more probably, that these residuals triggered the generation of such peculiar inflammatory response in a likely predisposed woman. In fact, many women are exposed to meconium and amniotic fluid at the time of cesarean section, and the majority of them do not develop such an atypical inflammatory reaction. A foreign body reaction is usually characterized by inflammatory cell infiltration of mainly macrophages with foreign body giant cells, fibroblast activation, and peripheral fibrosis [14]. Macrophages have specific roles, including M1 or M2 polarization and the consequent difference in the storage of collagen and fibrosis [15]. At this regard, it should be emphasized our decision to proceed with the immunofluorescence analysis of the pseudotumor tissue by flow cytometry. We performed this peculiar, but greatly belonging to 
our research expertise [7], assay specifically to understand the origin of the PET glucidic hypermetabolism and to better define the inflammatory component, considering that the intraoperative histology excluded the diagnosis of sarcoma. In our case, the analysis of the cell phenotype by flow cytometry demonstrated that M1-polarized Glut+ macrophages were the most dominant cell subsets. Therefore, the positive PET finding was certainly correlated to the high number of inflammatory cells, particularly CD14/Glut+ cells with high glucose uptake; meanwhile, the CD13+ and CD10+ cells were Glut-. These results confirm that the neoformation was an early granuloma that rapidly arose as a reaction to the spillage of meconium and keratinous material (vernix caseosa) present in the amniotic fluid in the subcutaneous tissue during cesarean section. The literature reports very rare cases of similar clinical conditions $[16,17]$, referred to as "organizing peritonitis, which included prominent collections of anucleate squamous cells in association with a foreign body-type granulomatous response" [16]. To our knowledge, this is the first case described in the literature.

\section{Conclusions}

The present paper provides information on an abdominal wall foreign body granuloma arisen from meconium and vernix caseosa after cesarean section, which is a unique and unpredictable condition that requires careful diagnosis and treatment. Different from sarcomas and desmoids, this atypical neoformation does not need a post-surgical follow-up and therefore it is essential to know how to diagnose it and not confuse it with other similar conditions. Thus, also atypical problems should be considered during the diagnostic and therapeutic phase emphasizing strongly a multidisciplinary approach to better planning its appropriate management.

\section{Abbreviations}

$\mathrm{CT}$ : Computed tomography; PET: Positron emission tomography; SUV: Standardized uptake value

\section{Acknowledgements}

The authors would like to thank Ivan Collu, Andreea Voicu, and Antonio Loffreda for their technical assistance. The work is supported by the

"Associazione Sarda per la ricerca in Oncologia Ginecologica-ONLUS."

\section{Availability of data and materials}

The datasets used and analyzed during the current study are extracted from the hospital clinical chart and are available from the corresponding author on reasonable request.

\section{Authors' contributions}

$\mathrm{AM}$ and $\mathrm{CM}$ contributed to the protocol development, data collection and management, data analysis, and manuscript writing/editing. PK, FL, GC, EM, and LM contributed to the data collection and management, data analysis, and manuscript editing. All authors read and approved the manuscript.

\section{Ethics approval and consent to participate}

Written informed consent was obtained from the patient for the surgical and medical approach.

\section{Consent for publication}

Written informed consent was obtained from the patient for the publication of the case report and the accompanying images.

\section{Competing interests}

The authors declare that they have no competing interests.

\section{Publisher's Note}

Springer Nature remains neutral with regard to jurisdictional claims in published maps and institutional affiliations.

\section{Author details}

1Department of Gynecologic Oncology, Azienda Ospedaliera Brotzu, via Edward Jenner, 09121 Cagliari, Italy. ${ }^{2}$ Department of Pathology, Azienda Ospedaliera Brotzu, Cagliari, Italy. ${ }^{3}$ Department of Nuclear Medicine, Azienda Ospedaliera Brotzu, Cagliari, Italy. ${ }^{4}$ Department of Public Health and Medical Sciences, University of Cagliari, Cagliari, Italy.

Received: 20 June 2018 Accepted: 26 September 2018

Published online: 10 October 2018

\section{References}

1. Stojadinovic A, Hoos A, Karpoff HM, Leung DH, Antonescu CR, Brennan MF, Lewis JJ (2001) Soft tissue tumors of the abdominal wall: analysis of disease patterns and treatment. Arch Surg 136:70-79

2. Levy AD, Manning MA, Miettinen MM (2017) Soft-tissue sarcomas of the abdomen and pelvis: radiologic-pathologic features, part 2-uncommon sarcomas. Radiographics 37:797-812

3. Gurluler E, Gures N, Citil I, Kemik O, Berber I, Sumer A, Gurkan A (2014) Desmoid tumor in puerperium period: a case report. Clin Med Insights Case Rep 7:29-32

4. Har-Shai Y, Govrin-Yehudain J, Ullmann Y et al (1993) Dermatofibrosarcoma protuberans appearing during pregnancy. Ann Plast Surg 31:91-93

5. Ozel L, Sagiroglu J, Unal A, Unal E, Gunes P, Baskent E, Aka N, Titiz MI, Tufekci EC (2012) Abdominal wall endometriosis in the cesarean section surgical scar: a potential diagnostic pitfall. J Obstet Gynaecol Res 38(3):526-530

6. Macciò A, Chiappe G, Kotsonis P, Lavra F, Serra M, Demontis R, Madeddu C (2017) Abdominal leiomyosarcomatosis after surgery with external morcellation for occult smooth muscle tumors of uncertain malignant potential: a case report. Int I Surg Case Rep 38:107-110

7. Madeddu C, Gramignano G, Kotsonis P, Coghe F, Atzeni V, Scartozzi M, Macciò A (2018) Microenvironmental M1 tumor-associated macrophage polarization influences cancer-related anemia in advanced ovarian cancer: key role of Interleukin-6 [published online April 19, 2018]. Haematologica. https://doi.org/10.3324/haematol.2018.191551

8. Dietz UA, Spor L, Germer CT (2018) Management of mesh-related infections Chirurg 82:208-217

9. Meagher H, Clarke Moloney M, Grace PA (2015) Conservative management of mesh-site infection in hernia repair surgery: a case series. Hernia 19:231-237

10. Narkhede R, Shah NM, Dalal PR, Mangukia C, Dholaria S (2015) Postoperative mesh infection-still a concern in laparoscopic era. Indian J Surg 77(4):322-326

11. Skubitz KM (2017) Biology and treatment of aggressive fibromatosis or desmoid tumor. Mayo Clin Proc 92:947-964

12. Wu Y, Chen L, Scott PG, Tredget EE (2007) Mesenchymal stem cells enhance wound healing through differentiation and angiogenesis. Stem Cells 25: 2648-2659

13. Basu S, Nair N, Banavali S (2007) Uptake characteristics of fluorodeoxyglucose (FDG) in deep fibromatosis and abdominal desmoids: potential clinical role of FDG-PET in the management. Br J Radiol 80:750-756

14. Williams GT, Williams WJ (1983) Granulomatous inflammation--a review. J Clin Pathol 36:723-733

15. Shapouri-Moghaddam A, Mohammadian S, Vazini H, Taghadosi M, Esmaeili SA, Mardani F, Seifi B, Mohammadi A, Afshari JT, Sahebkar A (2018) Macrophage plasticity, polarization, and function in health and disease. J Cell Physiol 233(9):6425-6440

16. George E, Leyser S, Zimmer HL, Simonowitz DA, Agress RL, Nordin DD (1995) Vernix caseosa peritonitis. An infrequent complication of cesarean section with distinctive histopathologic features. Am J Clin Pathol 103:681-684

17. Stuart OA, Morris AR, Baber RJ (2009) Vernix caseosa peritonitis - no longer rare or innocent: a case series. J Med Case Rep 3:60 